\title{
Exploring the Application of Artificial Intelligence in Sports Training: A Case Study Approach
}

\author{
Shiqing Wei, ${ }^{1}$ Puquan Huang, ${ }^{2}$ Rui Li, ${ }^{3}$ Zhiguo Liu, ${ }^{4}$ and Yuepei Zou (D) ${ }^{5}$ \\ ${ }^{1}$ Xiamen Xingcai Vocational and Technical College, Xiamen, Fujian 361000, China \\ ${ }^{2}$ Shenyang Sport University, Shenyang, Liaoning 110000, China \\ ${ }^{3}$ Chengdu College of Arts and Sciences, Chengdu, Sichuan 610401, China \\ ${ }^{4}$ Jose Rizal University, Manila 0900, Philippines \\ ${ }^{5}$ Zhengzhou University, Zhengzhou, Henan 450000, China \\ Correspondence should be addressed to Yuepei Zou; zouyp@zzu.edu.cn
}

Received 7 April 2021; Accepted 4 May 2021; Published 13 May 2021

Academic Editor: Huihua Chen

Copyright ( $\odot 2021$ Shiqing Wei et al. This is an open access article distributed under the Creative Commons Attribution License, which permits unrestricted use, distribution, and reproduction in any medium, provided the original work is properly cited.

\begin{abstract}
With the rapid development of computer science and information technology, artificial intelligence (AI) has been developed from theory to application. As a key technology in the modern society, AI is increasingly affecting all aspects of people's daily lives, including sports training. AI can be considered as an assistive technology to provide specific support to athletes' physical education training through various means such as data analysis and simulation of training scenarios. Although research on AI is still in the preliminary stage, it is significant to explore how AI can be applied in sports training since this emerging technology could facilitate people's physical training to some extent. This paper first reviews the existing research on AI applications. Then, based on the fundamental concept and related research results of AI, this study explores three specific cases of AI application in sports training and explains the main principles. This research focuses on discussing the strong relationship between AI technology and physical education training and highlights the advantages of AI, including utilization, convenience, and innovation.
\end{abstract}

\section{Introduction}

As a discipline created in the 1950s, artificial intelligence (AI) is defined as the ability of a system to interpret and learn from exterior data correctly and to adopt the learning results to achieve specific objectives and solve problems through flexible adaptation [1]. It belongs to a branch of computer science that researches how computers could learn, think, and reason [2]. Based on this, Housman proposed that AI owns two abilities: one is to generate repetitive assignments by predicting the outcome of categorical data, and the other is to make human-like decisions by dealing with issues with algorithms [3]. In recent years, with the continuous development of global technology, intelligent technology is also developing rapidly. As a member of the emerging technology, AI has long been applied to various fields, such as voice recognition, image recognition, driverless technology, and so on. In a short period, AI has turned from theory to practical application and has provided more job aids for humans and has even begun to partially replace human work. After all, AI is machine or computer intelligence which is able to carry specific tasks for human such as diagnosing diseases [4]. Thus, AI can be regarded as a way to reduce costs and improve the quality of services, cooperation, and productivity $[5,6]$. In addition, AI can assist humans in decision analysis from a theoretical perspective. Machine learning belongs to a subfield of AI, and it can provide decision-making analyses for people. It contains a large variety of advanced algorithms. For instance, BP neural network algorithm can be applied to solve the issue of optimizing the allocation of taxi resource at the airport [7]. Cheng et al. constructed a system dynamic model based on machining learning to imitate the influence of government policies on prefabricated construction and this would be 
helpful to improve policies [8]. Yang et al. adopted the Markov chain algorithm to forecast the freight volume in Xi'an, and it can provide the relevant departments with some useful strategies [9]. Therefore, the application of AI is significant and wide.

Currently, AI has also emerged in the field of sports. It has the potential of sustainable development in physical education training as long as the fundamental theoretical structure appropriate for this goal is built when integrating with other fields. As in most other areas of society, an increasing amount of data is being collected in different physical activities, and the utilization of AI for automated data analysis has become an important research direction. Since traditional statistical methods are slow and inaccurate, automated data analysis with AI has been widely used. AI can simulate human learning, thinking, perception, and actions using advanced computer algorithms and actually the AI system is self-learning $[10,11]$. In the development of computer science, AI is gradually becoming more advanced and automated. AI is capable of finishing automated tasks more quickly and effectively as well as making better decision than humans [12]. Hence, it is able to analyze data and obtain valuable information just like humans, but AI can handle large amounts of data that humans cannot. Analyzing physical activity data can deepen the human exploration of sports science and improve the sports personnel's decisionmaking ability in optimizing physical training and competitive strategies. In conclusion, enhancing the related studies of AI may be beneficial to lay the foundation for the modern physical education field. Therefore, it is necessary and essential to apply AI to physical education training.

Physical education technology belongs to many fields such as sports, education, and computer science [13]. At the same time, the rapid development of science and technology is influencing the educational modes and types of educational systems significantly [14]. As a typical representative of the development of science and technology, AI can effectively assist people in physical education training. It is relatively difficult to analyze the various data generated during training accurately without equipment in the conventional sports training. Hence, some imprecise data might affect people's physical education training negatively. However, AI could be beneficial to cope with practical issues and assist in physical training when developing physical education technology. For instance, to help athletes improve their performance in competitive sports, the right approaches and a great deal of data are needed. The technical analysis includes motion capture, data acquisition, and data study. AI can extract and analyze data from a large number of training sessions of athletes, which would lead to corresponding valuable conclusions and suggestions. Therefore, AI has a certain application value in the field of sports training.

In order to better apply AI technology to this area, many studies have been conducted to explore the integration of computer technology with the physical movement of the human body. For example, Lei developed a badminton technical feature statistics and pace training system, which can achieve two major functions of badminton game technical statistics and player's pace training based on the badminton action recognition algorithm [15]. The result indicates that the accuracy rate is $96.7 \%$, which means that the system can accurately measure the athletes' pace information and help to quickly improve their pace levels. Liang applied the support vector machine (SVM) to construct an evaluation model of taekwondo teaching effectiveness based on an AI algorithm [16]. The model enables correct students' motion by identifying their taekwondo movement characteristics and can instruct them scientifically through simulation methods. Du designed a youth physical exercise system based on AI technology which is composed of an object inspection module, a data analysis module, and a body posture determination module, and this system can be applied to estimate human posture by establishing posture-assisted indicators [17]. The experiment result indicates that this system based on AI can have a positive impact on improving teenagers' physical quality and providing scientific training methods for them. Sohn and Choi used 10 Vicon cameras, 2 AMTI force plates, and an AI-based analysis system to calculate the striking abilities of 5 various golf balls and found the factors that can influence the power of golf shots [18]. Goksel and Bozkurt introduced an expert system based on AI which is used to own the same ability of dealing with issues as experts after inputting their knowledge and experience into a computer [19]. This system can be applied in the sports field because it can also provide some professional suggestions about training.

The above examples show that if the data of athletes' movements and various physical indicators can be grasped in real time during physical training, then the corresponding instructional plans can be gained through computer processing, and timely feedback can be provided for better physical training. However, previous research has not systematically investigated the integrated application of AI in physical education training. Although the previous studies are specific, they lack a more complete summary of the application of AI in sports training. Also, there has been little research to explore the application of AI to physical education training although AI is significant in improving the sports training system in the future. Therefore, according to the main concepts and principles of AI, this study explores the various implementations of AI on facilitating people's physical training and provides several real cases to analyze the main application of AI. Meanwhile, the principles of each case analysis are revealed to better explain how AI can achieve scientific and efficient work.

\section{Analysis of Wearable AI Devices}

2.1. Introduction of Wearable AI Devices. Wearable smart sports products refer to digital products that can take the form of accessories or clothing. They are designed to use modern information networks and various sensor technologies to record various vital data to monitor the body's overall physical state [20]. The emergence of wearable smart sports products is the result of people's demand for scientific exercise and product intelligence. Therefore, it will become a major breakthrough in a new field to improve people's 
quality of life and integrate sports activities and artificial intelligence.

The design model of wearable AI devices comes from the Internet development. The hardware core is a variety of physiological information sensors and wearable technologies, while the core technologies at the software level are wireless network transmission and statistical data processing. The technology incorporates sensors, multimedia and wireless communication technologies to enable sensing, and feedback and interactive experiences for basic human body movements. It can collect human physiological parameters during the whole physical training process and can provide athletes with feasible advice through a big data analysis system. For example, smart wearable watches can accurately measure users' specifics in exercise such as the heart rate (Figure 1). The most significant advantage of smart wearable watches is their portability. In the past, health testing professionals used large and stationary devices to collect data on the user's physical characteristics, but the disadvantage is that less data is collected and it is not easy to detect occasional situations. Thus, the appearance of wearable AI devices can be an efficient way to fill this gap.

The Wearable Devices Research Report released by the China Academy of Information and Communication Technology points out that the market size of smart wearable products in China has reached 12.5 billion yuan in 2015, with a growth rate of an astonishing $471 \%$, and the rapid development of wearable artificial intelligence products from the world has been the trend of the development of the Internet of Things. Therefore, wearable AI devices have a quite broad market prospect and huge development potential in the field of modern physical education training.

2.2. Case Analysis of Wearable AI Devices. The MySwing Professional is a golfer training auxiliary equipment that can accurately capture the player's movement and the club's trajectory based on whole-body movement analysis. It is an AI-based product designed and developed by the Noitom company. This device includes 17 wireless full-body sensor nodes and connected wearable retractable straps with builtin wireless antennas and preinstalled MySwing software for real-time playback and cloud storage, which is shown in Figure 2. The precise positioning system can capture the player's movements accurately since the player wears multiple micromotion sensors. At the same time, there is also a sensor near the handle of the club, which could be used to record the spatial movement data of the club as well as provide the analysis results. Then, it can adopt many different data visualization methods to help players as well as coaches to observe the technical details more efficiently.

The MySwing Professional has three main functions to achieve the effect of AI technology completely: motion capture, playback analysis, and storage comparison. The specific details of each function are shown below.

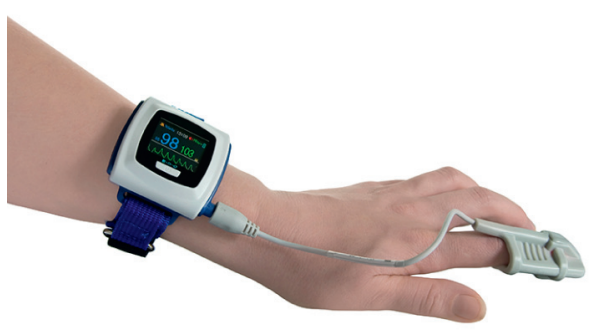

Figure 1: Smart wearable watches.

2.2.1. Motion Capture. Before starting, the player first puts on a full-body wireless motion capture suit and simply calibrates it; then, MySwing Professional would record the player's movements in precise details. Actually, the function of motion capture is achieved by sensors worn throughout the player's body and sensors on the club that record every movement with precision. The sensors are key to realize this function since the data collected from the sensor coordinate frame can be transformed to the computer by estimating the sensor orientation [21].

2.2.2. Playback Analysis. Quantifying sports data is crucial to the game of golf because the analysis of data can help players improve their movements. In response to this need, MySwing Professional provides a comprehensive solution. Players can not only observe their swings in a 3D environment in real time but also analyze the angle and acceleration of their swings through playback, making the state of motion easier to grasp. Since the 3D model, analysis chart, and other auxiliary reference tools have the characteristics of data visualization, players can observe and analyze their own swings from a comprehensive perspective and compare them with more skilled professional golfers. Therefore, this function is quite essential to the improvement of player's ability.

2.2.3. Storage Comparison. The MySwing Professional will upload a golfer's movement data from every session to a cloud-based server, so they can keep track of their level changes through the data and compare it to the movement data of professional golfers. This function is reasonably practical and useful to players since each of their exercises can be stored, and then they can use these records to review the details of their previous movements.

Actually, MySwing Professional is already in use and has been adopted by many famous players in their daily training. For example, DeChambeau, a champion professional golfer in the United States, utilizes MySwing Professional to watch his shots from all angles and compare them side by side with other professionals' movements on record to find out where his movements are lacking and continuously adjust to his best level. 


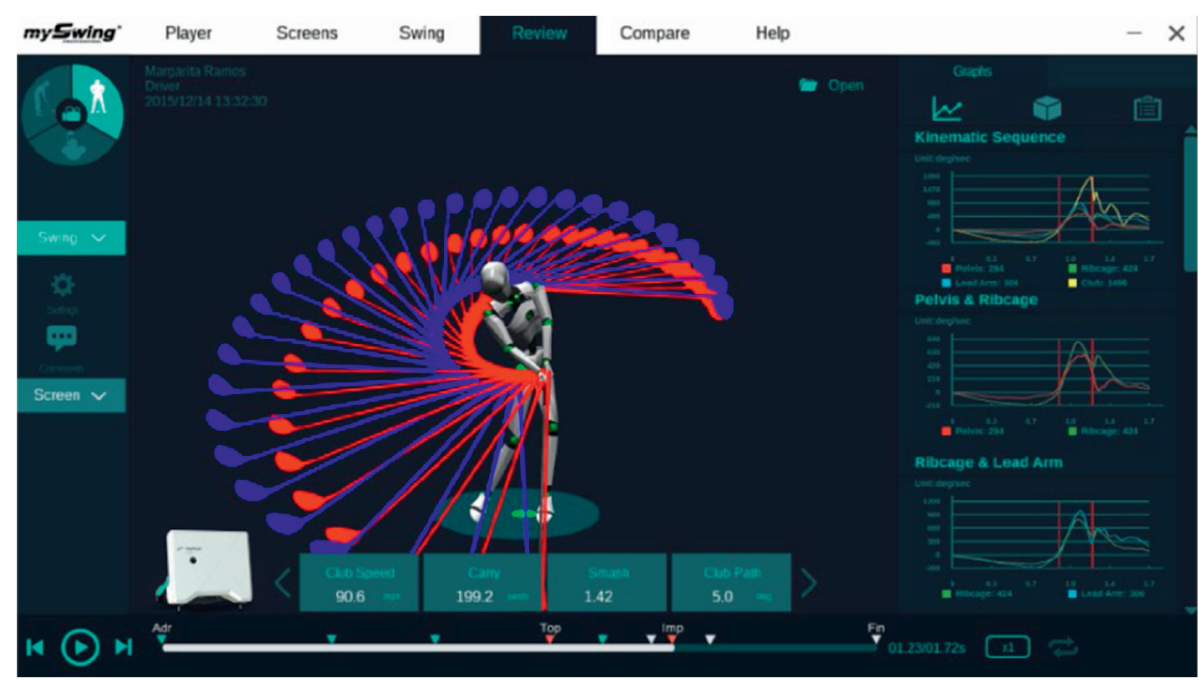

Figure 2: Real-time playback and cloud storage functions of MySwing Professional.

In summary, MySwing Professional has many advantages such as convenience and intelligence in practical use. It can help players to perform better in their daily training in order to improve their relevant skills. Thus, it is a successful example of combining AI with physical education training.

\section{Analysis of Visual Target Tracking System Based on AI}

3.1. Introduction of Visual Target Tracking System Based on AI. Real-time tracking of athletes' movement and their position as well as targeted coaching plans are vital in physical education training [22]. It can help athletes to analyze their performance in sports training more intuitively. Hence, the visual target tracking system has been an important research direction in the field of AI in recent years. The primary purpose of developing the system is to enable computers and humans to communicate smoothly and to understand human speech, gestures, and other actions that can go further than the human brain can accomplish [23]. This system uses cameras and computers to capture, track and measure targets, and realize automatic action recognition through AI algorithms, breaking many limitations of traditional action recognition technology.

The visual target tracking system mainly consists of four parts: target detection, target recognition, target processing, and target display (Figure 3).

Firstly, the visual target tracking system can detect the trajectory of the target's movement by using an image detection module. This module is mainly composed of cameras that could capture the image of the target. In order to accurately obtain the target's position, posture, and other information, it is necessary to install the corresponding cameras around the target following a specific topology to obtain the target's image information in real time. In this part, the topology is essential because the topology between different regions performs a key role in obtaining various visual relationships [24]. The placement of the camera can generally use a wrap-around structure to ensure that the

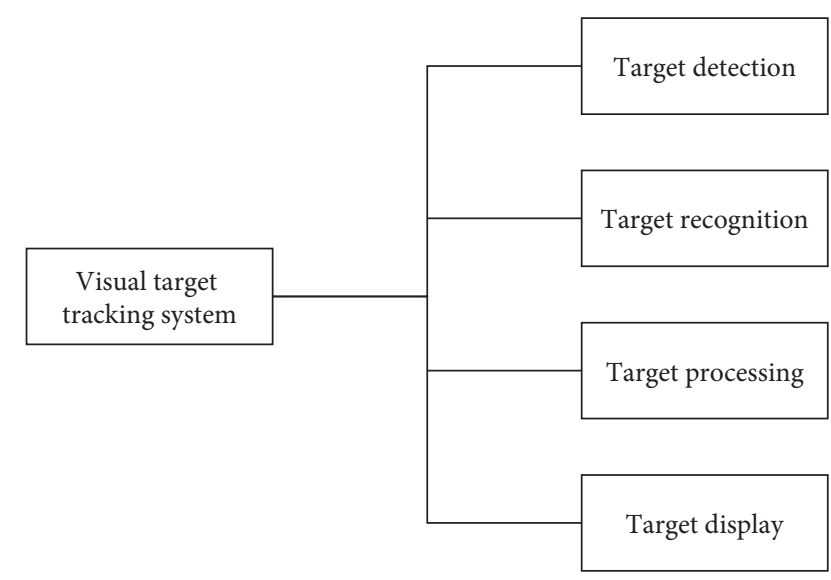

FIGURE 3: Main parts of visual target tracking system.

target's movement information can be obtained in all directions, thus providing accurate input information to the system. Then, this image is transported to the target recognition part. In this part, the image can be recognized by the image recognition module which includes a computer processing system. The computer is able to run some complex AI algorithms to recognize the state of the target. After that, this computer can process this image to analyze the movement state of target. Image recognition in this part is quite important in the system because it can turn the complicated motion into data analysis [2]. Finally, the analysis result will be shown as images or data to people intuitively. Hence, the visual target tracking system could realize motion analysis of the target.

3.2. Case Analysis of Visual Target Tracking System Based on AI. In team sports, the tactical coordination and choice of tactics between athletes is an important factor in determining the game. Therefore, it is necessary to continuously track and analyze the athletes' movements during the competition so that they can be better trained during the 
day. The technical and tactical analysis methods vary from sport to sport, but the basic steps are similar. Firstly, the raw data is collected, and then the valid information is extracted. The last step is to analyze the data in depth. In most official competitions, athletes are not allowed to wear additional equipment, so the information collection method is mainly visual based. Hence, the visual target tracking system based on $\mathrm{AI}$ can be applied in this situation.

SportVU is an intelligent basketball game analysis system that has been widely applied in NBA. It uses six cameras suspended from the ceiling of the arena to track players and basketballs to analyze every dribble, pass, distance to teammates, and distance run during the game (Figure 4). The system can acquire 25 images per second to track and analyze the player's movement. Behind the camera are also connected various sensors for dynamic capture, trajectory analysis, data extraction, and then importing the processed data into the database. In addition to providing traditional data records, the system can also answer many complicated questions that traditional data analysts cannot answer. Therefore, SportVU can transform previously unquantifiable game information into a collection of data that can be further mined and applied using machine learning methods to help team data analysts and coaches gain better insight into the inner workings of their teams. In conclusion, this system provides an alternative way of complementing and even replacing the traditional coaching methods to some extent.

Before the start of each basketball game, the SportVU operator has to set up the system, which includes setting the sensors to follow the players, marking the court boundaries, and assigning individual player profiles to the detected objects. The process is as follows:

(1) Three high-definition cameras or sensors are placed at the console, each recording one-third of the playing field.

(2) The live video is recorded into the computer system, where the lines of sight of the three cameras converge to form the playing field.

(3) Any object on the field (player, referee, and ball) appears as a dot on the operator's computer screen. The software can track players' movements through these dots.

The SportVU system could track every player's movement on the court, and the increased access to information has led to a significant increase in the amount of data to be processed. However, SportVU users generally believe that the proportion of data used is less than $10 \%$ of the total data provided by the system, and the importance of developing AI technologies to exploit the value of the data better is further highlighted. Thus, some AI algorithms are vital to decipher the patterns of movement behind the data. For example, in the case of a pick-and-roll, the system can identify different screens and determine whether the screener is going to cut in after the screen or cut out after the screen and calculate the success rate for each tactic. All of

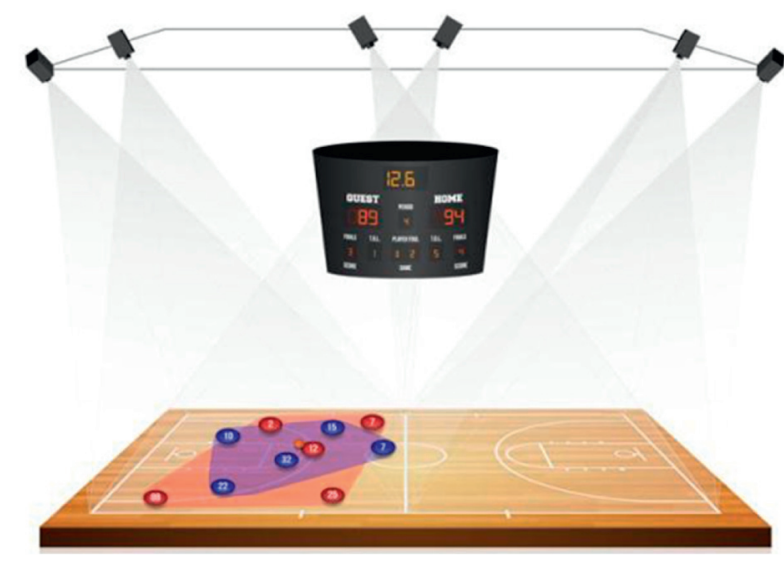

FIgURE 4: SportVU system.

this is based on AI algorithms to achieve this important function.

In conclusion, as a typical application of a visual target tracking system, SportVU could accurately capture athletes' movements as well as process and analyze large amounts of data by using AI algorithms. Based on this intelligent system, the coaches are able to master athletes' information in sports in time and provide some practical training plans which are suitable for them. With this visual target tracking system, the athletes can also train more specific to their own characteristics. Hence, the visual target tracking system based on AI plays a vital role in physical education training in the modern society.

\section{Analysis of Virtual Reality Technology}

4.1. Introduction of Virtual Reality Sports Simulation System. Virtual reality (VR) is an emerging technology that brings together multiple fields such as computer graphics, sensing technology, and AI technology [25]. It integrates multimedia, AI, human-machine interface technology, dynamic device network, and some other information technologies to simulate a true world by building a virtual world [26]. VR technology is a computer simulation system to simulate a virtual network space so that the user is immersed in a virtual environment with three-dimensional information. In this virtual environment, users could realize the real-time interaction of multiple senses which are considerably real. In the three-dimensional dynamic scene created by a computer simulation system, based on the head-mounted display, data gloves, intelligent language, and other interactive devices, users can achieve visual, auditory, and tactile multisensory enhanced experience feedback. This technology has characteristics of interactivity, immersion, and reality which can enable people to feel the reality in the virtual network.

The VR simulation system includes multiple perceptual and is interactive and immersive. At the same time, physical education training requires the joint participation of multiple senses of athletes such as vision, hearing, and touch. Therefore, with the continuous development of VR 
technology, VR-based simulation has been widely used in physical education training (Figure 5). VR has become one of the most significant and effective tools for education and training [27]. It can provide athletes with effective and new training methods which can help them to improve their skills. Also, for some group sports' training programs, VR can deal with problems such as insufficient numbers of teammates to better meet their training needs. Therefore, VR is becoming more increasingly popular with people who want to have a better training experience in recent years.

The following functions are necessary to VR-based sports' simulation system.

4.1.1. Building Virtual Training Scenarios. Some sports such as basketball have special requirements for training venues, so virtual training scenarios and virtual training equipment need to be modeled. In other words, this function is the most fundamental part in the VR training system. It can simulate the real training scenes, thus providing the most basic virtual training scenes for athletes. In this virtual environment, athletes will feel as if they are training in the real world, which means that they would own a great training scenario. Meanwhile, this virtual environment allows them to train even though they do not have a real training field.

4.1.2. Capturing Motion Data. The system can provide sensor tracking devices to record data about the athlete directly and analyze it with a computer for simulation. When the athletes are training, the specific devices can automatically store their data such as heart rate and movement speed. The most important feature is that it could capture data from real human movements. The data of athletes in training is quite essential since they can summarize and overview their training state through the data. Therefore, the function of capturing motion data is a necessary part in the VR-based sports simulation system.

4.1.3. Collection of Physiological and Psychological Data. Physiological and psychological indicators are important reflections of the athletic state of the athlete. Depending on the type of sport, the physiological and psychological data of athletes can be collected by various sensors. For instance, the collection of physiological data includes metabolic indicators such as pulse rate, blood pressure, and functional indicators of various organs. The collection of psychological data contains the athletes' mood fluctuations in training.

4.1.4. Action Replay. Action replay is the most significant function of the system. The traditional camera methods cannot work well under some conditions. Some training methods cannot be realized because they are in the innovative stage, but VR can deal with this issue. For example, in gymnastics, coaches can help athletes improve and innovate their movements and improve their skills by modeling the

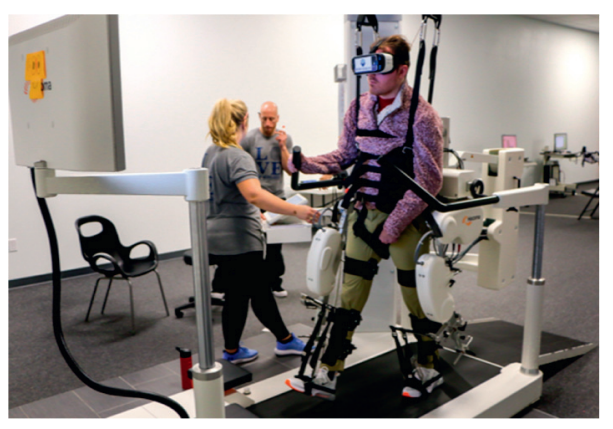

FIgURE 5: VR-based simulation system.

movements of real athletes, creating new movements, and then using VR to simulate the new movements.

The VR sports simulation system can be divided into immersive and nonimmersive systems. The immersive systems require helmet-based 3D stereo displays, stereo glasses, data gloves, stereo headsets, high-performance computers, and some other equipment. These devices allow users to experience realistic stereo vision and stereo hearing to interact with the virtual environment. The nonimmersive system mainly relies on software technology to create a virtual world with rich visual and auditory information. To sum up, the characteristic of immersive systems is expensive but strongly immersive, while the characteristic of nonimmersive systems is cheap and conventional.

4.2. Case Analysis of Virtual Reality Sports Simulation System. QB SIM is a professional training simulation system based on VR, which can be used for daily repetitive training of football players (Figure 6). This system allows football quarterback training to be conducted efficiently in a safe, realistic virtual reality environment, helping players grow quickly out of high school and into the National Football League (NFL). In other words, this system could assist users in better training.

The QB SIM simulation training system is built in a real rugby stadium, and by combining OptiTrack motion capture technology and VR virtual reality technology, athletes can get the most realistic training experience. The football itself is a rigid body with motion capture marker points on its surface, and the headset worn by the athlete is also equipped with marker points on its surface. The QB SIM system then uses motion capture technology to obtain the athlete's positions and the football and set the movements and play of the virtual player in the game, thus enabling efficient training.

By using the QB SIM simulation training system to assist with training, athletes can train more easily when faced with practical problems. For example, it is known that the football game is a team game. Namely, it becomes less realistic if the athletes still wish to train when there are not enough people to train. Nevertheless, the QB SIM simulation training system could enable them to train without other teammates 


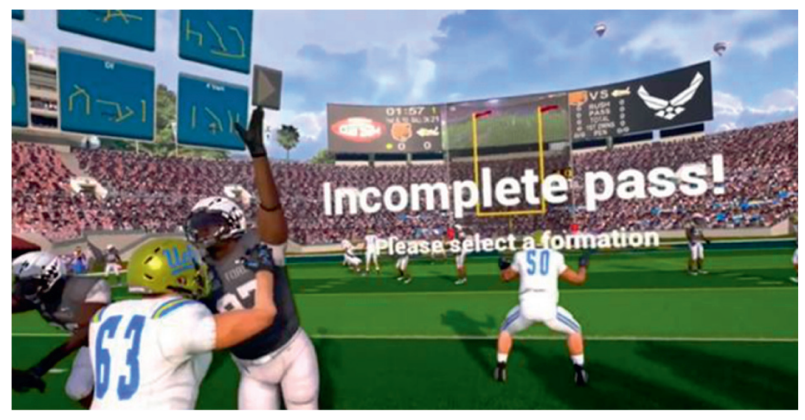

FIGURE 6: QB SIM simulation training system.

because it can create many virtual people to train with the user.

\section{Conclusion}

This paper provides an introduction to the use of AI technology in physical education training and highlights three practical examples. The cases are analyzed carefully through explaining their principles and functions, respectively. Firstly, this study introduces the wearable AI devices and takes MySwing Professional as an example to illustrate the successful application of AI in the golf game. Then, the main parts of the visual target tracking system based on AI are described, and SportVU is chosen to suggest that this system has been widely used in the basketball game of basketball. Finally, the virtual reality sports simulation system is introduced and QB SIM is a typical application of the system in the football game. Thus, the three practical applications of combining AI technology with physical education training indicate that the AI technology can better enhance the traditional sports training. AI could provide precise data analysis and scientific plans which will improve the training efficiency for athletes. Also, some devices based on AI can create virtual training environment, and it is quite conventional for athletes to train. In terms of the impact of our research on practice, it should be noted that innovations in AI are affecting various areas of people's lives. Therefore, the implementation of AI in other areas should be discussed more. In conclusion, as a new and rapidly developing technology, AI technology will affect more and more people in the future.

\section{Data Availability}

All data generated or used during the study are available within the article.

\section{Conflicts of Interest}

The authors declare that they have no conflicts of interest regarding the publication of this paper.

\section{References}

[1] A. Kaplan and M. Haenlein, "Siri, siri, in my hand: who's the fairest in the land? on the interpretations, illustrations, and implications of artificial intelligence," Business Horizons, vol. 62 , no. 1, 2018.

[2] P. Ding, "Analysis of artificial intelligence (AI) application in sports," Journal of Physics Conference Series, vol. 1302, 2019.

[3] M. Housman, "Why "augmented intelligence" is a better way to describe AI. AI news," 2018, https://www. artificialintelligence-news.com/2018/05/24/why-augmentedintelligence-is-a-better-way-to-describe-ai/.

[4] S. Russell and P. Norvig, Artificial Intelligence: A Modern Approach, Prentice Hall, Upper Saddle River, NJ, USA, 2nd edition, 2003.

[5] T. H. Davenport, The AI Advantage: How to Put the Artificial Intelligence Revolution to Work, MIT Press, Boston, MA, USA, 2018.

[6] M. Iansiti and K. Lakhani, Competing in the Age of AI: Strategy and Leadership when Algorithms and Networks Run the World, Harvard Business Review Press, Boston, MA, USA, 2020.

[7] Y. M. Qian, S. H. Chen, J. C. Li et al., "A decision-making model using machine learning for improving dispatching efficiency in Chengdu Shuangliu airport," Complexity, vol. 2020, Article ID 6626937, 16 pages, 2020.

[8] B. Q. Cheng, Y. H. Wei, W. Zhang et al., "Evolutionary game simulation on government incentive strategies of prefabricated construction: a system dynamics approach," Complexity, vol. 2020, Article ID 8861146, 11 pages, 2020.

[9] F. Yang, X. Y. Tang, Y. X. Gan, X. D. Zhang, J. C. Li, and $\mathrm{X}$. Han, "Forecast of freight volume in Xi' an based on gray GM $(1,1)$ model and Markov forecasting model," Journal of Mathematics, vol. 2021, Article ID 6686786, 6 pages, 2021.

[10] I. Roll and R. Wylie, "Evolution and revolution in artificial intelligence in education," International Journal of Artificial Intelligence in Education, vol. 26, no. 2, pp. 582-599, 2016.

[11] R. L. Adams, 10 Powerful Examples of Artificial Intelligence in Use Today, Forbes, Jersey City, NJ, USA, 2017, https://www. forbes.com/sites/robertadams/2017/01/10/10-powerfulexamples-of-artificial-intelligence-in-use-today/ \#55427b67420d.

[12] H. S. Lee and J. Lee, "Applying artificial intelligence in physical education and future perspectives," Sustainability, vol. 13, 2021.

[13] L. Xian, "Artificial intelligence and modern sports education technology," in Proceedings of the 2010 International Conference on Artificial Intelligence and Education, pp. 772-776, Hangzhou, China, October 2010.

[14] D. McArthur, M. Lewis, M. Lewis, and M. Bishary, "The roles of artificial intelligence in education: current progress and future prospects," i-manager's Journal of Educational Technology, vol. 1, no. 4, pp. 42-80, 2005.

[15] Y. C. Lei, Research and Implementation of Badminton Technical Characteristics Statistics and Pace Training Based on Machine Learning, Central China Normal University, Wuhan, China, 2018.

[16] H. L. Liang, "Role of artificial intelligence algorithm for taekwondo teaching effect evaluation model," Journal of Intelligent and Fuzzy Systems, vol. 40, no. 2, pp. 3239-3250, 2021.

[17] C. Du, “Assistant training system of teenagers' physical ability based on artificial intelligence," Mathematical Problems in Engineering, vol. 2021, pp. 1-10, 2021.

[18] J. Sohn and H. Choi, "Are golf-shots distinguished by power control? or it is just individual differences?" International Journal of Performance Analysis in Sport, vol. 13, no. 1, pp. 212-224, 2013. 
[19] N. Gksel and A. Bozkurt, "Artificial intelligence in education: current insights and future perspectives," 2019.

[20] L. Shi, Y. M. Wang, H. Hu, H. M. Wang, and X. T. Peng, "Application of wearable artificial intelligence products in modern physical education," 2018.

[21] A. Yurtman and B. Barshan, "Activity recognition invariant to sensor orientation with wearable motion sensors," Sensors, vol. 17, no. 8, p. 1838, 2017.

[22] B. Ma, S. Nie, M. Ji, J. Song, and W. Wang, "Research and analysis of sports training real-time monitoring system based on mobile artificial intelligence terminal," Wireless Communications and Mobile Computing, vol. 2020, no. 6, pp. 1-10, 2020.

[23] B. G. Baumgart, "A polyhedron representation for computer vision," in Proceedings of the American Federation of Information Processing Societies: National Computer Conference, Anaheim, CA, USA, May 1975.

[24] L. Zhang, Y. Wang, H. S. Chen, J. Li, and Z. X. Zhang, "Visual relationship detection with region topology structure," Information Sciences, vol. 564, 2021.

[25] C. Y. Jie, C. H. Chen, and C. J. Ming, "Integrating videocapture virtual reality technology into a physically interactive learning environment for English learning," Computers \& Education, vol. 55, no. 3, pp. 1346-1356, 2010.

[26] H. S. Lee and Y. K. Jin, "A review of benefits and trends for the three specific and distinct products using technology in physical education," Journal of Korean Association of Physical Education \& Sport for Girls \& Women, vol. 30, no. 4, 2016.

[27] S. Baek, S. Lee, and G. J. Kim, "Motion retargeting and evaluation for VR-based training of free motions," The Visual Computer, vol. 19, no. 4, pp. 222-242, 2003. 